\title{
Preparing for Blockchain Technology in the Energy Industry: How Energy Sector Leaders Can Make Informed Decisions During the Blockchain Adoption Process
}

\author{
Haideh Farahmand \\ Athabasca University \\ hfarahmand1@athabasca.adu
}

\author{
Arta Farahmand \\ Athabasca University \\ afarahmand1@athabasca.edu
}

\begin{abstract}
This research was motivated by the lack of literature about the constructs influencing the decision to adopt blockchain technology. This paper contributes to the knowledge by integrating common adoption and diffusion theories with a 2017 framework for blockchain adoption. This paper brings together competing adoption models with different sets of technology acceptance determinants and proposes a new model to identify constructs (i.e., ease of understanding, perceived usefulness, the perceived ease of use, knowledge acquisition, self-efficacy, and the novelty and complexity of the new technology application) as essential determinants of blockchain technology adoption at individual and organizational levels. The study offers a new model and research agenda to help executives and managers prepare for blockchain adoption and make informed decisions to speed up the adoption process. This research is focused on energy companies, which are known to be slow to adopt new technologies.
\end{abstract}

\section{Introduction}

Although blockchain technology is perceived to be disruptive, there is no clear grasp of where and how the technology can be effectively applied [25]. That is why blockchain technology is sometimes considered "an innovative technology in search of use cases" [13, p. 1543], and sometimes judged to be over-hyped [25]. Current research on blockchain falls into the following categories: features and design of the technology, measurement of value, and organization/management research. So far, research has been primarily centered on the features and design of the technology, while research on value and organization/management are scarce [25]. However, with interest in blockchain technology on the rise, attention to organizational/management research in this field is growing; for example, a recent study proposes a research agenda centered on governance, looking at decision rights, accountability and incentives in the new blockchain economy [5]. The gaps in organization/management on blockchain research motivated this study. Considering the importance and size of the energy industry, this research started by investigating sensible use cases for blockchain technology in the energy sector. Then the study expanded to explore how the energy industry can prepare for blockchain technology adoption.

Oil and gas companies are known to be slow in adopting new technologies [9]; however, analysts report that oil and gas industry executives are closely monitoring the new developments in blockchain technology to evaluate the potential impact and disruptions of this innovative technology [12]. Digital solution providers such as ConsenSys are actively developing blockchain-based solutions for the petroleum industry [9]. It is worth noting that the proliferation of technologies such as the Internet of Things (IoT) in the oil and gas industry has transformed the amount and kinds of data now collected. Data transformation in the industry increasingly requires a vessel to carry it forward. Blockchain could be that vessel [16].

Worldwide desire to increase renewable energy generation has drawn attention to the distributed nature of renewable energy sources. Geographically distributed electricity generation demands innovative approaches to connect energy producers with consumers. Recently, blockchain technology has created excitement as a platform that enables peer-topeer exchange of electricity [22] (for more information about peer-to-peer energy transactions, refer to [18]).

This paper focuses on blockchain adoption in two key energy industry subsectors: oil and gas industry and renewable energy. In the oil and gas industry, blockchain has significant potential to manage data transformation, given the data-intensive opportunities enabled by new technologies such as IoT [16]. In 
renewable energy generation, geographically distributed electricity generation calls for intelligent connection among suppliers; this can be enabled by blockchain [22]. Thus innovations in the electricity grid and in oil and gas organizations can be considered major activities in energy-focused blockchain development [8].

The goal of this paper is to identify key constructs that determine decision to adopt blockchain technology at individual and organizational levels. This study combines Iansiti and Lakhani's 2017 proposed framework for blockchain adoption [15], in connection with common adaptation and diffusion theories: Social Cognitive Theory (SCT) [3], Innovation Diffusion Theory (IDT) [26], and Technology Acceptance Models (TAM [11] \& TAM2 [34]). The research findings are consolidated as a new model for blockchain adoption (Figure 1). A research agenda is then proposed to evaluate the new model and test whether adoption constructs derived from literature actually explain blockchain adoption.

This study's findings make a practical contribution by enabling executives and managers to speed up blockchain adoption, in that their decisions about the adoption process can be informed by an understanding of the key constructs that determine blockchain adoption. These constructs can be influenced or controlled to increase acceptance and accelerate the adoption of blockchain technology. The findings are particularly important for the energy sector due to its history of slow adoption of new technologies [9].

This paper starts with a brief introduction to benefits of blockchain technology for the energy industry, followed by a short description of how blockchain works and some of the challenges to large-scale adoption of blockchain technology. Then, the common adoption and diffusion theories are explored [3, 26, 11, 34], followed by examination of Iansiti and Lakhani's 2017 framework for blockchain adoption [15]. Finally, the paper integrates adoption decision determinants from common theories with the blockchain adoption framework, and proposes a new model and research agenda for blockchain adoption.

\section{Background and rationale}

This section explores the major opportunities for innovation and improving efficiency in the energy sector through the adoption of blockchain technology.

One of the strongest applications of blockchain technology in the oil and gas industry is energy trading. Blockchain technology provides a reliable and efficient platform for trading energy by executing transactions through smart contracts (contracts governed by a decision algorithm deployed on the blockchain) and recording transactions on a digital, distributed ledger that provides all parties with synchronized and simultaneous access to the information. Blockchain technology allows ownership to be tracked even if assets change hands a number of times before the final settlement [12]. Smart contracts thus transform relationships with vendors and suppliers, and significantly reduce the probability of disputes. Blockchain also enhances efficiency by simplifying processes. By offering instant verification of transactions across a network (without the necessity for verification by a central authority), blockchain has the potential to reduce operating cost, speed up transactional processing, and store and manage data more securely [16]. Supply chain management and finance activities will be improved [12].

Another important trend is the increasingly distributed nature of electricity generation since the emergence of renewable energy as a growing source for power generation. The emergence of smart grids, which enable two-way communication between the utility and its consumers [36], requires peer-to-peer energy trading as an energy management mechanism [30]. In this energy revolution, utilities and consumers both produce and sell energy, and blockchain is increasingly being used in utility and power companies as a low cost and reliable way to facilitate these peer-to-peer transactions [4].

\subsection{Challenges to large-scale blockchain adoption}

This section describes some of the challenges associated with the large-scale adoption of blockchain across industries, and then recounts some of the barriers associated with the implementation of blockchain in the energy sector.

Blockchain systems currently face a number of technical issues that impede large-scale adoption of the technology, such as capacity and limited query capabilities compared to other distributed database systems. However, since these limitations are likely temporary [13], the description of technical issues is excluded from this analysis, and this paper instead focuses on non-technical factors that impede adoption of the technology, as described in the practical literature.

Forbes magazine in September 2017 identified the highly technical nature of blockchain technology, which makes it hard to describe its value, as one of the barriers to large-scale adoption. Words such as "mining" and "crypto" can be confusing, and people will not adopt blockchain if they don't understand its value [35]. Most of the terminology used to describe blockchain was coined by the cryptographers who came up with the 
technology; however, cryptographers are not necessarily marketers, and most people haven't been able to come into terms with many of the new concepts. How can the concept of a block and the idea of creating a new block be explained to a non-technical audience? How can the concept of a node and role of miners be explained to executives in organizations that are considering adoption of the blockchain technology [35]? Another challenge managers face is to learn enough about the technical aspects of blockchain to make decisions. Executives looking for blockchain technology partners for their organizations have to consider multiple options, some of them viable, and some not [24]. How can they judge viability if they don't understand the technology?

Furthermore, almost all blockchain applications are non-consumer-friendly. For example, nearly all call for users (organizations adopting blockchain technology) to either run a node or install a light node [1], a level of technical ability that businesses may find intimidating. Other obstacles include the following:

- high cost, which is a significant barrier to mass adoption of blockchain;

- the locked-in nature of blockchain platforms (the code and infrastructure that secure the blockchain removes the ability to change the platform if it proves suboptimal for the specific application; and

- the limited features, which do not meet the high expectations for blockchain technology created by the news media [1].

At the organizational level, other obstacles exist. Companies face a challenge when integrating blockchain into their legacy systems. For example, an organization might use a CRM system (Customer Relationship Management) to manage relationships with customers, a provisioning system to provide access to technology resources and data, and a billing system to fulfill orders. Data storage models and formats can be different for all these systems. Most of these systems may work in isolation and may have limited integration with other business systems. Thus, organizations need to carefully evaluate their business operations and understand how blockchain architecture can be redesigned for each use case [17] to connect with existing systems.

Government regulation is another barrier to implementing blockchain in the energy industry. The oil and gas industry is highly regulated, and the fluid nature of regulation relating to blockchain makes implementing the technology a risk at this time [10]. Furthermore, the peer-to-peer transactions would require a redefinition of the current regulatory framework for the energy sector before utilities could adopt blockchain. A blockchain-based market model calls for changed market roles [7].
Another challenge for the oil and gas industry is privacy concerns about the distributed ledger, which carries data from different companies. The fact that data from different companies is transparent to all users (members of a public or consortium blockchain) raises anti-trust and collusion concerns. There is also the risk of being compromised by hackers [10]. Moreover, the proliferation of blockchain players and platforms (e.g., Ethereum, Hyperledger, Ripple, etc.) and system configurations (e.g., public, private, and hybrid) for different applications of the technology poses a risk for the interoperability of these systems [10].

\subsection{How blockchain works}

Blockchain is defined as a distributed ledger. It can be described as a different way of storing records of transactions [6]. At its simplest, blockchain is a database of such records. The system enables users to exchange tokens within the blockchain network. A "transaction" is the transfer of tokens from one user to another, which requires the data in this database to be updated [13]. The database keeps track of exactly who owns which tokens, at each step of the trade.

The name of the technology, blockchain, refers to the organization of these transactions as a chain of blocks [14]; transactions and records are batched in blocks [2], which are linked together in chains. From a technical standpoint, a blockchain consists of multiple nodes that can be distributed across the globe and are connected through peer-to-peer communication. A node can be any electronic device that has an IP address and is connected to the Internet [13]. Parties who don't trust each other can use blockchain to conduct and reliably control transactions without using services of a trusted third party [25].

Blockchain records are encrypted for protection against manipulation. All transactions within each block are stored in a cryptographic data structure [5]. Each user has a public key and a private key. A user's private key cryptographically signs transactions and messages [13].

The core functionality of blockchain technology is to enable transactions that are validated and immutable. Blockchain can enable consistent database updates across a global network of nodes [13].

Blockchain uses consensus mechanisms to assure database consistency when a new transaction is validated. Consensus mechanisms are used to incentivize nodes across the blockchain to validate new transactions; often through economic incentives [5]. The most common consensus mechanisms are proof-ofwork and proof-of-stake [21, 6]. Proof-of-work consensus is based on solving cryptographic puzzles. The first node that solves the puzzle will validate the 
next block and will receive the economic incentive with cryptocurrency [5]. However, proof-of-work consensus mechanisms require massive amounts of energy. For example, Bitcoin's proof-of-work mechanism is estimated to consume as much energy as a country like Switzerland in one year [19]. Proof-of-stake solves the high energy consumption problem [29]. In proof-ofstake, having more cryptocurrency leads to higher probability for the nodes to be selected to validate the next block. If the node behaves maliciously, the stakes may be destroyed [5] (for more information on consensus mechanisms, refer to [29]).

Current generations of blockchain technology, such as Ethereum and Hyperledger, feature a built-in programming language and a virtual machine for execution of the programs. The codes in blockchain are deployed at every node and specific transaction events can trigger them. This feature is significant because it facilitates far more applications of the blockchain technology than simply cryptocurrency transactions. These codes are called smart contracts [13].

Blockchains can be categorized according to whether authorization is required for nodes to participate in the verification process (permissioned blockchains), or anyone can participate as a node to verify blocks (permissionless blockchains). In permissioned blockchains, a central authority selects verification nodes [23]. Furthermore, blockchains can be classified based on whether blockchains are public, meaning that everyone can submit transactions and gain access to the information, or private, where access to the blockchain is restricted and only users with permission (e.g., members of a group or organizations) can submit transactions and access information [23].

It is worth noting that until now, the Internet has enabled peer-to-peer exchange of information; however, blockchain enables peer-to-peer exchange of values (real assets). That is why blockchain is referred to as an "internet of values" [28, p. 4].

\section{Theoretical framework}

This section reviews the common theories that provide insight into the key constructs that determine the decision to adopt a new technology.

Challenges associated with a positive reaction to new technologies have been a key topic of information system research [31]; the adoption and utilization of new technological advancements is a concern of information system researchers [32, 34]. There is much at stake: proper implementation of technology adoption can improve efficiency, while ineffective implementation can result in discontent and loss [32].
Adoption and diffusion theories are concerned with the process involved in disseminating a new idea over time. No single model provides an understanding of individual adoption determinants [27]. It is important to recognize the difference between adoption and diffusion theories. Adoption involves behavior change (an individual's decision to adopt or resist an innovative technology) and can be predicted through affective, cognitive and contextual elements. However, diffusion is a process of collective adoption over time [27]. Diffusion theories describe how the perception of an innovative technology spreads among people [27].

This paper explores Social Cognitive Theory (SCT) by Bandura [3], Innovation Diffusion Theory (IDT) by Rogers [26], and the Technology Acceptance Models (TAM \& TAM2) by Davis [11] and Venkatesh and Davis [34] respectively. The goal is to use these theories to identify key constructs that determine the decision to adopt a new technology. Furthermore, a framework for the adoption of blockchain technology, proposed by Iansiti and Lakhani in 2017 [15], will be examined to identify additional constructs that can impact blockchain adoption decision.

Social cognitive theory posits that knowledge is an important determinant of innovation adoption; however, knowledge acquisition and skills are not sufficient for individuals to make the decision to adopt a new technology [3]. Other factors such as incentives and environmental stimulus are considered regulators in making adoption decisions [3]. Social cognitive theory uses three processes to analyze the diffusion of new behavioral patterns: first, the process of acquiring knowledge about the innovative behaviors; second, the process of adopting these behaviors in practice; and third, the process through which social networks spread and support the innovation [3]. At the beginning, an innovation spreads slowly because of people's lack of knowledge and familiarity with it, their resistance to change, and the uncertainty of achieving the desired outcome by adopting the innovation. As the knowledge spreads and the results are demonstrated by early adopters, the rate of adoption increases. In this period, adoption spreads swiftly. However, after a while, depending on the value experienced by adopting the innovation, use either stabilizes or declines [3].

The speed of adoption is influenced by mechanisms and psychological determinants of observational learning. Observational learning is controlled by attention, retention, production, and motivation processes [3]. Attention processes include the innovation's attractiveness and functional value, and are influenced by structural interactions and social networks. Retention processes affect the speed of adoption and involve transforming information into concepts that reinforce memory representation. 
Production processes involve the translation of cognitive symbolic concepts into appropriate actions. Developing capabilities and continuously improving them through corrective actions is part of production processes. Finally, motivational processes recognize that people don't necessarily act after acquiring knowledge. The incentives that motivate action include direct and vicarious incentives such as values experienced directly or by others, as well as selfproduced incentives such as personal values [3].

Ease of understanding and self-efficacy (the belief that one can successfully apply the new technology to one's tasks) are determinants in the decision to acquire and adopt a new technology. Self-efficacy can be achieved by using the technology in simple applications that generate positive results, which will then create the motivation for adoption [3].

According to the Innovation Diffusion Theory (IDT), diffusion is a form of communication that disperses ideas over time across a population. IDT examines the decision to adopt an innovation at both individual and organizational levels. At the individual level, the adoption decision is not quick; it is a process. IDT considers diffusion and adoption processes as inseparable, and recognizes five stages in the process of evaluating an innovation:

1) Awareness of the innovation is impacted by personal characteristics (e.g., change-seeking personalities), level of interaction with change agents (e.g., media) and socioeconomic factors (e.g., the individual's work experience, social and economic position, etc.).

2) Persuasion occurs when a person's knowledge about the innovation's important characteristics grows to the point that the individual forms a judgement (either favorable or unfavorable) toward the innovation.

3) Decision occurs when the person chooses whether to reject or adopt the innovation.

4) Implementation of the innovation takes place when the individual takes action based on his or her decision.

5) Confirmation happens when a person reflects on his or her decision about the adoption and implementation of the innovation and revisits the whole process. At this stage, the individual decides whether to continue using the innovation, or to stop and discontinue the adoption process [26].

It is important to note that individuals can only make an adoption decision when their organizations have decided to adopt a technology [26]. For organizations to make an adoption decision, key players must believe that the innovation fits the problem in the organization. There are five stages in an organization's adoption process:
1) identifying problems that have the potential to be perceived as creating a need for the innovation;

2) matching a problem from the potential list with the innovation;

3) adjusting the innovation to fit the organization's need, and restructuring the organization accordingly;

4) improving understanding of how the innovation relates to the organization's needs; and

5) turning the innovation into a routine activity [26]. Organizational decisions about adopting new technologies are made at higher levels; however, individual adoption plays a key role in successful implementation of new technologies [27].

The Technology Acceptance Model (TAM) posits that ease of use is a significant factor in the acceptance of new technology $[11,31]$. The perception of control over the technology after one has learned about it, motivation resulting from the playfulness of the new technology, and emotional anxiety about adopting the new technology all regulate the perception of ease of use [32]. The Technology Acceptance Model 2 (TAM2) postulates that the perceived usefulness is a construct that determines system adoption. Factors in considering the technology's usefulness include its job relevance, perceptions about its ease of use, the quality of the outputs delivered by the technology and the ability to demonstrate the results. Other factors include image, voluntariness and subjective norms. TAM2 encourages the implementation of new systems through social influence, in contrast to compliance-based adoption [34]. It is important to emphasize that TAM2 supports creating a suitable organizational context for the adoption of a new technology.

Constructs described in this section as determinants of individual and organizational adoption (i.e., ease of understanding, perceived usefulness, perceived ease of use, knowledge acquisition and self-efficacy) can be applied to blockchain technology adoption and provide insight into the proper interventions (decisions to increase acceptance and effective use of the technology) for streamlining the implementation process.

In addition to the common diffusion and adoption theories, Iansiti and Lakhani's 2017 framework for blockchain adoption describes how a foundational technology such as blockchain takes hold [15]. The framework distinguishes between the adoption of a disruptive technology, which changes an established business model by adopting more cost-effective solutions, and the adoption of a foundational technology, which can impact the societal and economic state of the world [15]. The model posits that, during the implementation of a foundational technology, there is a need to recognize the novelty of the technology's application, as well as the complexity of the 
coordination effort needed to make the application workable. Applications that are low in novelty and complexity are accepted first. However, applications that are high in novelty and complexity make take decades to evolve and be accepted [15]. Blockchain can be considered a foundational technology.

Iansiti and Lakhani's framework describes the impact of novelty and complexity on blockchain adoption in the context of four phases for the foundational technology to take hold:

1) Adopting single-use applications that are low in novelty and complexity: These applications are the easiest place to start and pose low risk, as they minimize third-party involvement and are not new. Bitcoin is an example of single-use application of blockchain technology.

2) Adopting localized applications: Localized applications are somewhat high in novelty but can generate value with a limited number of users (parties in the blockchain network), which means that these applications are low in complexity (less intensive coordination/governance is required if there are only a few members in the blockchain network). An example of localized application is a private blockchain application to establish local private networks that enables multiple organizations to collaborate through a distributed ledger to address particular needs.

3) Adopting substitution applications: In a substitution application, current ways of doing business are replaced by the new foundational technology (e.g., blockchain). Such applications are based on the technology's established pattern of single-use and localized applications. Substitution applications are not new (i.e., low novelty); however, because of public use, these applications must be highly coordinated (i.e., high complexity). These applications face more obstacles during adoption, not only due to a high need for coordination, but also because the processes they aim to replace are deeply embedded within organizations. Cryptocurrencies are examples of the substitution application of blockchain technology, as they challenge governments by changing the payment method for transactions.

4) Adopting transformational applications: Transformational applications have the potential to alter economic, societal and political landscapes. These applications require massive coordination to gain consensus on standards and processes. An example of the transformational application of blockchain technology is the use of smart contracts, in which a protocol (a decision algorithm) digitally enforces the terms and conditions of the contract without the need for third-party oversight, and automatically releases payments and transfers currency once the conditions of the contract are met [15].

\section{Findings/Discussion}

In this section, findings from exploring Social Cognitive Theory (SCT) [3], Innovation Diffusion Theory (IDT) [26], Technology Acceptance Models (TAM [11] and TAM2 [34]), and Iansiti and Lakhani's blockchain adoption framework [15] are reviewed to identify the determinants governing the adoption of blockchain technology. (Identifying the relationships between these determinants is beyond the scope of this paper and is part of the research agenda put forward by this study.)

Venkatesh and Bala [33] assert that, as the complexity of the information technology system increases, low adoption rates present a more serious problem. Thus, it becomes more important to enable managers to make informed decisions to intervene and increase technology acceptance and the use of IT systems [33]. This study assumes that the same logic applies to blockchain technology and addresses how energy sector executives and managers can prepare for blockchain adoption. The assumption is that by understanding the key constructs that influence people and organizations to adopt a new technology, executives and managers can speed up the adoption process through informed decisions, building on knowledge about the determinants of blockchain technology adoption.

According to SCT, ease of understanding and selfefficacy are determinants of the adoption decision [3]; this aligns with IDT, which identifies knowledge acquisition about the innovation as the first step in the individual adoption decision [26]. It worth noting that SCT details the impact of attractiveness, functional value, and social networks on the speed of blockchain adoption, and emphasizes the importance of making visible the direct and vicarious value of the technology in order to motivate users [3].

According to IDT, technology adoption at the individual level starts by acquiring knowledge about the new technology, which leads to an attitude that influences the decision to adopt [26]. At the organizational level, variables such as the technical expertise of employees, the organizational structure (i.e., centralization of power in the organization) and the availability of slack resources influence organizational innovativeness [26], which in turn influences the adoption decision. The finding from exploring SCT and IDT is that they both recognize knowledge acquisition as a key determinant of the adoption decision. However, IDT outlines five stages in an individual's adoption of 
new technology (awareness, persuasion, decision, implementation, confirmation); this suggests that knowledge by itself is not sufficient for individuals to make the decision to adopt something new. According to IDT, the second stage of individual's evaluation of innovation is persuasion. Straub argues that any organization that is considering implementation of an innovative technology must create processes to deal with affective, cognitive and contextual aspects of the adoption process [27]; this can be done through strategic executive decisions and tactical decisions during managers' implementation of the technology. This study assumes that informed decisions during the adoption process can be made to positively influence individuals' attitudes toward the technology, which according to IDT happens prior to the adoption decision (third stage).

Furthermore, ease of understanding and selfefficacy, posited by SCT as determinants of technology adoption, align with IDT as they influence the second stage of an individual's evaluation of an innovative technology (persuasion). This study assumes that selfefficacy and ease of understanding influence individuals to form a positive attitude towards the technology.

In addition, this study assumes that the perception of ease of use (TAM) and the perception of usefulness (TAM2), both will influence second stage of the individual's evaluation of an innovative technology (persuasion) to address affective, cognitive, and contextual concerns.

The analysis of the theoretical findings points to the importance of managerial actions following individuals' awareness of a new technology, in order to influence individuals' perceived ease of use, which can lead to self-efficacy, and the perceived usefulness of the technology, which according to TAM2 can address users' cognitive concerns. Furthermore, ease of understanding can influence users to acquire the level of knowledge that facilitates persuasion.

Based on this analysis, this study suggests that at the individual level, adoption decisions can be influenced by knowledge acquisition, ease of understanding, selfefficacy, perceived ease of use and perceived usefulness. These constructs regulate individuals' emotional, cognitive and contextual reactions to a new technology.

At the organizational level, IDT theory points to the available slack resources in organizations as a variable that influences organizational innovativeness [26], which aligns with Iansiti and Lakhani's framework [15]. The novelty and complexity of blockchain applications influence the amount of resources required to facilitate technology adoption, and thus influence organizations adoption decision.
Theoretical findings align with observations reported in practical publications. For example, as described earlier in this paper, understanding the technology and the value it creates influences the adoption decision [35]. Furthermore, recognizing that current blockchain applications are not consumer friendly (a barrier to adoption) [1] aligns with perceived ease of use as a construct that influences the adoption decision.

These findings advance theory and contribute to the future research on blockchain adoption behavior. Based on the theoretical grounding, this paper proposes a new model for blockchain adoption, as depicted in Figure 1. The proposed model can be useful to energy sector executives and managers, especially given increasing investment in blockchain technology in this sector. The proposed model helps leadership to prepare for blockchain technology by acquiring knowledge about determinants of blockchain technology adoption.

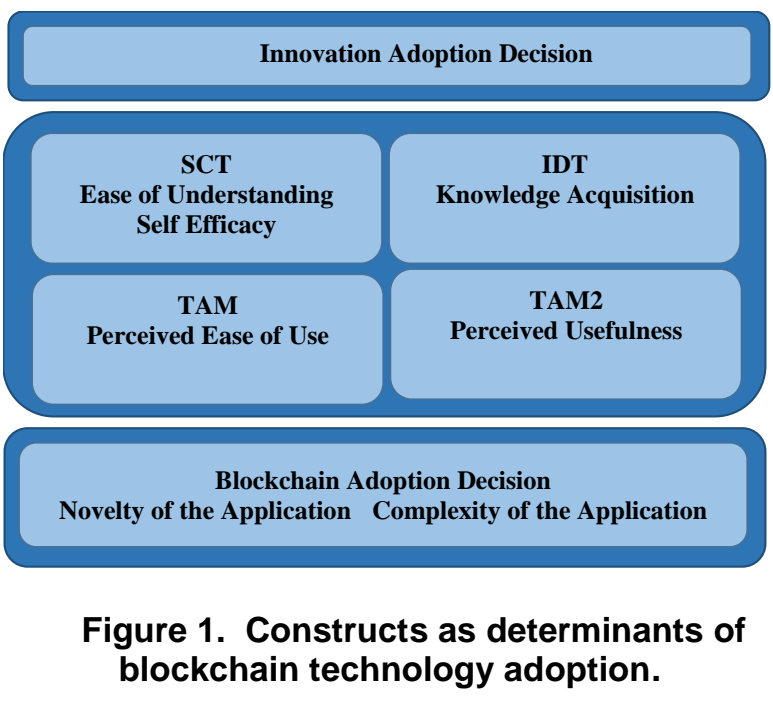

\section{Methodology/Hypothesis}

This conceptual paper is based on review and analysis of two streams of literature. First, the literature review focused on exploring whether blockchain technology can be effectively applied in the energy sector with material impact: would it increase efficiency or disrupt the industry through novel business models? In light of the opportunities presented in the literature, the second phase of the literature review focused on adoption and diffusion theories to determine how the energy industry can prepare to adopt blockchain technology. The literature points to constructs that influence the blockchain adoption decision. The findings were presented in the form of a new model (Figure 1) to present these determinants. 
The hypotheses in this study are: 1) there is a correlation between the adoption of blockchain technology and the following constructs: the ease of understanding, perceived usefulness, perceived ease of use, knowledge acquisition, and self-efficacy, as well as the novelty and complexity of the coordination effort needed to make the application workable ; 2) there is a correlation between executives' and managers' understanding of the constructs that influence blockchain adoption, and their ability to make decisions to speed up adoption during the technology implementation process.

The causalities in the proposed model is defined as executives' and managers' ability to make informed decisions to expedite blockchain adoption as a dependent variable, and the level of knowledge about the constructs that influence blockchain technology adoption (ease of understanding, perceived usefulness, perceived ease of use, knowledge acquisition and selfefficacy, as well as the novelty and complexity of the new technology application) as an independent variable.

The next section proposes a research agenda to advance knowledge about the determinants of blockchain adoption.

\subsection{Research agenda}

Further empirical research is required to evaluate the proposed model and test whether the constructs derived from the theoretical literature actually explain blockchain adoption. Future research should measure and analyze how each construct: ease of understanding, perceived usefulness, perceived ease of use, knowledge acquisition and self-efficacy, as well as the novelty and complexity of the new technology application influences the adoption decision.

Similarly, research is needed to analyze the relationships among the constructs derived from adoption and diffusion theories: ease of understanding, perceived usefulness, perceived ease of use, knowledge acquisition and self-efficacy.

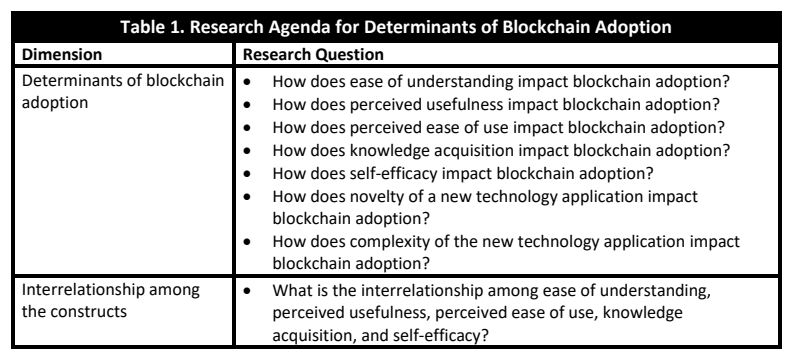

\section{Conclusion}

Academic studies of blockchain have primarily focused on design and technology. Research on measuring the value of the applications of blockchain in various industries and organization/management research is scarce [25]. The goal of this study was to explore the potential applications and value of blockchain technology in the energy sector, and, considering the opportunities identified in the literature, provide an answer to the question: how can the energy sector prepare for blockchain technology?

In order to address this question, common adoption and diffusion theories - Social Cognitive Theory (SCT) [3], Innovation Diffusion Theory (IDT) [26], and Technology Acceptance Models (TAM [11] and TAM2 [34]) — were examined to identify the determinants of the decision to adopt a new technology. The findings were integrated with additional determinants for blockchain adoption that were identified in the 2017 framework proposed by Iansiti and Lakhani for blockchain adoption decision [15]. A new model was proposed to identify the determinants of the blockchain adoption decision (Figure 1).

This study was motivated by the energy sector's increasing interest in blockchain technology [9, 20], and the history of slow adoption of technological advancements in the energy sector [9]. The assumption is that knowing the determinants of blockchain adoption can help energy sector executives and managers to make informed decisions to speed up the adoption process.

This study briefly described blockchain technology and its value for two key energy industry subsectors: the oil and gas industry and the renewable energy industry. Blockchain presents significant potential in oil and gas [16] and renewable energy [22] industries, and the major activities in the energy blockchain space can be classified as innovations in the electricity grid, and innovations in oil and gas organizations [8].

As part of the study, non-technical challenges to large-scale implementation of blockchain technology were explored by researching practical publications to verify alignment of the findings with the theoretical determinants of blockchain adoption.

This paper's contribution to the theoretical literature is to integrate adoption and diffusion theories to identify constructs that influence the blockchain adoption decision. This paper brings together multiple competing adoption models with different sets of technology acceptance determinants, in order to identify the key determinants of blockchain adoption at the individual and organizational level. The study findings contribute to academic discussion of the variables that influence the adoption decision for blockchain technology.

The practical contribution of this paper is to propose an abstract model to help executives and managers understand the constructs that influence individuals' 
decision to adopt blockchain technology, thus enabling informed decisions by executives and managers to increase the technology's acceptance and to improve blockchain utilization. The significance of this finding for the energy industry is that it will help energy companies deal with potential resistance and slow adoption patterns. Knowledge about the determinants that influence the adoption process helps executives with decision making and can lead to greater acceptance and more effective use of blockchain technology, and can speed up the adoption process.

Finally, an agenda was presented for future research to evaluate the proposed model and explore the interrelationship between the constructs (ease of understanding, perceived usefulness, perceived ease of use, knowledge acquisition and self-efficacy).

\section{References}

[1] 5 Obstacles Currently Facing Blockchain Technology, Inc. BrandView, 2018, retrieved from: https://www.inc.com/arcblock/five-obstacles-currentlyfacing-blockchain-technology.html

[2] Appelbaum, D., and S. Stein Smith, "Blockchain Basics and Hands-on Guidance: Taking the Next Step toward Implementation and Adoption", CPA Journal, 88(6), 2018, pp. 28-37.

[3] Bandura, A., "Social Cognitive Theory of Mass Communication", Media Psychology, 3(3), 2001, pp. 265299.

[4] Basden, J., and M. Cottrell, "How Utilities Are Using Blockchain to Modernize the Grid [White Paper],", Harvard Business Review, 2017, retrieved from: http://www.oliverwyman.com/content/dam/oliverwyman/v2/publications/2017/may/How_Utilities_Are_Using Blockchain_To_Modernize_The_Grid.pdf

[5] Beck, R., C. Müller-Bloch, and J. L. King, "Governance in the Blockchain Economy: A Framework and Research Agenda", Accepted in March 2018 for publication in the Journal of the Association for Information Systems, retrieved from:

file://C:/Users/haideh/Downloads/Governanceintheblockchai neconomyaframeworkandresearchagenda\%20(1).pdf

[6] Beckage, J., "Understanding the Practical and Legal Challenges of Blockchain", Rochester Business Journal, 33(24), 2017, pp. 23-24, retrieved from: http://0search.proquest.com.aupac.lib.athabascau.ca/docview/19422 $\underline{31506 ? \text { accountid }=8408}$

[7] Blockchain - An Opportunity for Energy Producers and Consumers?, PwC Global Power \& Utilities 2016, retrieved from: https://www.pwc.com/gx/en/industries/assets/pwc- blockchain-opportunity-for-energy-producers-andconsumers.pdf

[8] Blockchain and the Energy Industry: An Overview of the Current State of the Energy Industry's Exploration and Integration of Blockchain Technology, Consensys, 2018, retrieved from: https://media.consensys.net/the-state-ofenergy-blockchain-37268e053bbd

[9] "Blockchain Technology Officially Enters the Oil and Gas Industry," CCn, February 10, 2018, retrieved from: https://www.ccn.com/blockchain-technology-officiallyenters-the-oil-and-gas-industry-with-ondiflo/

[10] Cann, J., "Blockchain - Facing up to the risks for oil and gas", Digital Oil and Gas, February 5, 2018, retrieved from: http://digitaloilgas.com/blockchain-facing-up-to-the-risksfor-oil-and-gas/

[11] Davis, F.D., "Perceived Usefulness, Perceived Ease of Use, and User Acceptance of Information Technology", MIS Quarterly, 13(3), 1989, pp. 319-339.

[12] Gain, B., "The Oil and Gas Industry Wakes Up to Blockchain", Rigzone, December 14, 2017, retrieved from: https://www.rigzone.com/news/the_oil_and_gas_industry_w akes_up_to_blockchain-14-dec-2017-152805-article/

[13] Glaser, F., "Pervasive Decentralization of Digital Infrastructures: A Framework for Blockchain Enabled System and Use Case Analysis", Proceedings of the $50^{\text {th }}$ Hawaii International Conference on System Sciences, Hawaii, U.S., 2017.

https://scholarspace.manoa.hawaii.edu/bitstream/10125/4133 9/1/paper0190.pdf

[14] Halaburda, H., "Economic and Business Dimensions Blockchain Revolution without the Blockchain?", Communications of the ACM, 61(7), 2018, pp. 27-29, doi:10.1145/3225619.

[15] Iansiti, M., and K. R. Lakhani, "The Truth About Blockchain [White Paper]", Harvard Business Review, January-February 2017, retrieved from: https://enterprisersproject.com/sites/default/files/the_truth_ab out_blockchain.pdf

[16] Koeppen, M., D. Shrier, and M. Bazilian, "Is Blockchain's Future in Oil and Gas Transformative or Transient?", Deloitte, 2017, retrieved from: https://www2.deloitte.com/content/dam/Deloitte/de/Docume nts/energy-resources/gx-blockchain-report-future-in-oil-andgas.pdf

[17] Kumar, P., "Blockchain: What Are the Implementation Challenges", IDG connect, March 22, 2018, retrieved from: https://www.idgconnect.com/blogabstract/29865/blockchain-what-implementation-challenges

[18] Lacey, S., "How Peer-to-Peer Energy Trading on the Blockchain Might Work [audio podcast]", gtm, 2018, 
retrieved

from:

https://www.greentechmedia.com/articles/read/how-peer-topeer-energy-trading-on-the-blockchain-might-

work\#gs.UMXr SA

[19] Lee, S. "Bitcoin's Energy Consumption Can Power an Entire Country - But EOS Is Trying to Fix That", Forbes, April 19, 2018, retrieved from: https://www.forbes.com/sites/shermanlee/2018/04/19/bitcoin s-energy-consumption-can-power-an-entire-country-but-eosis-trying-to-fix-that/\#117faf $921 \mathrm{bc} 8$

[20] Metelitsa, C., "4 Predictions for Blockchain in Energy in 2018", March 5, 2018, retrieved from gtm (A Wood Mackenzie Business): https://www.greentechmedia.com/articles/read/fourpredictions-for-blockchain-in-energy-in-2018\#gs.LaMeTgs

[21] Murray, J., "The Coming World of Blockchain: A Primer for Accountants and Auditors" CPA Journal, 88(6), 2018, pp. 20-27.

[22] NAD Grid, White Paper, March 26, 2018, retrieved from: https://www.nadgrid.com/wpcontent/uploads/2018/03/NADGrid-Whitepaper-032717$\underline{\text { v1.pdf }}$

[23] Peters, G. W., and E. Panayi, "Understanding Modern Banking Ledgers through Blockchain Technologies: Future of Transaction Processing and Smart Contracts on the Internet of Money", Banking Beyond Banks and Money, Springer International, 2015, retrieved from: https://arxiv.org/pdf/1511.05740v1.pdf.

[24] Radocchia, "What Are the Biggest Barriers to Creating Blockchain Companies", Forbes, March 2, 2018, retrieved from: https://www.forbes.com/sites/quora/2018/03/02/whatare-the-biggest-barriers-to-creating-blockchaincompanies/\#6c27dc8932f1

[25] Risius, M., and K. Spohrer, "A Blockchain Research Framework", Business \& Information Systems Engineering, 59(6), 2017, pp. 385-409.

[26] Rogers, E. M., Diffusion of Innovations, Free Press, New York, 1995.

[27] Straub, E. T., "Understanding Technology Adoption: Theory and Future Directions for Informal Learning", Review of Educational Research, 79(2), 2009, pp. 625-649, doi:10.3102/0034654308325896.
[28] Tapscott, D., and A. Tapscott, "Realizing the Potential of Blockchain: A Multistakeholder Approach to the Stewardship of Blockchain and Cryptocurrencies [White Paper]", World Economic Forum, 2017, retrieved from: http://www3.weforum.org/docs/WEF_Realizing_Potential_B lockchain.pdf

[29] Tschorsch, F., and B. Scheuermann, "Bitcoin and Beyond: A Technical Survey on Decentralized Digital Currencies", IEEE Communications Surveys \& Tutorials, 18(3), 2016, pp. 2084-2123.

[30] Tushar, W., C. Yuen, H. Mohsenian-Rad, T. Saha, H. V. Poor, and K. L. Wood, "Transforming Energy Networks via Peer to Peer Energy Trading: Potential of Game Theoretic Approaches", arXiv preprint arXiv:1804.00962, 2018, retrieved from Cornell University Library: https://arxiv.org/pdf/1804.00962.pdf

[31] Venkatesh, V., "Creating Favorable User Perceptions: Exploring the Role of Intrinsic Motivation", MIS Quarterley, 23(2), 1999, pp. 239-260.

[32] Venkatesh, V., "Determinants of Perceived Ease Use: Integrating Perceived Behavioural Control, Computer Anxiety and Enjoyment into the Technology Acceptance Model", Information System Research, 11(4), 2000, pp. 342-365.

[33] Venkatesh, V., and H. Bala, "Technology Acceptance Model 3 and a Research Agenda on Intervention", Decision Science, 39(2), 2008, pp. 273-315.

[34] Venkatesh V., and F. D. Davis, "A Theoretical Extension of the Technology Acceptance Model: Four Longitudinal Field Studies", Management Science, 46(2), 2000, pp. 186204.

[35] "What's Holding Blockchain Back from Large-Scale Adoption?", Forbes, September 21, 2017, retrieved from: https://www.forbes.com/sites/quora/2017/09/21/whatsholding-blockchain-back-from-large-scaleadoption/\#37d1d3b23092

[36] "What Is Smart Grid?”, Smartgrid.gov, n.d., retrieved from:

https://www.smartgrid.gov/the_smart_grid/smart_grid.html 